\title{
A Comparative Study of Drospirenone and Desogestrel: An Overview of Benefits and Side Effects
}

\author{
Parnamita Bhattacharya, Nandini Kapoor, Amit Kyal, Partha Mukhopadhyay
}

Calcutta Medical College and Hospital, Kolkata, India

\begin{abstract}
Aims: To study the antiandrogenic and antimineralocorticoid activity of drospirenone, a novel progestogen used as a component of combined oral contraceptive pill (COC) and compare it's clinical and biochemical effects with that of desogestrel, the most commonly used progestogenic component of COC.

\section{Methods}

Study design: prospective analytical study.

Population and setting: study was conducted in our department from July 2008 to June 2010 . Two hundred women were randomly allocated into two groups. First group received combination of $3 \mathrm{mg}$ drospirenone and $30 \mathrm{mcg}$ ethinyl estradiol (DRSP/EE) and the other group was given COC containing $150 \mathrm{mcg}$ desogestrel and 30 mcg ethinyl estradiol (other OCP group).

Results: The DRSP/EE group showed no significant decrease in body weight, Ferriman gallwey Score (FG), systolic and diastolic blood pressure. No evidence of sodium retention seen in any patients. $86 \%$ women had decrease in serum sodium from baseline. There was significant increase in HDL-Cholesterol level by $7.52 \%$ and no significant decrease in LDL-Cholesterol level. In the other OCP group, 52 subjects had increase in body weight, 30\% showed increase in FG score and none had any decrease in blood pressure. 62\% women had net gain in serum sodium level. Serum Triglyceride, HDL-Cholesterol, LDL-Cholesterol all were increased.

Conclusion: DRSP/EE combination is well tolerated, demonstrating good cycle control and a beneficial effect on skin condition. It exerts significant antiandrogenic activity and is effective in improving hirsutism. It also has positive effect on serum electrolyte composition and lipid profile.
\end{abstract}

Keywords: Combined oral contraceptives, drospirenone, desogestrel

\section{Introduction}

The oral contraceptive pill (OCP) is one of the most extensively studied medications ever prescribed. The health benefits are numerous and outweigh the risks of their use. Despite the efficacy and availability of OCP, many women who require continuing contraception, stop using it primarily because of tolerability issues including cycle control, weight gain, water retention, perimenstrual symptoms and hypertension ${ }^{1,2}$. Most common type of OCP used is the combined monophasic preparation containing $30 \mathrm{mcg}$ of ethinyl estradiol and $150 \mathrm{mcg}$ of desogestrel. Side effects due to the estrogen component are dose related. So, newer preparation of pills are formulated having lower dose of oestrogen but with equal contraceptive efficacy.
Importantly, the progestogenic component usually determines the overall metabolic and clinical effects, and therefore influences individual contraceptive choice $^{3}$. As a consequence, ongoing clinical trials have focused on developing more selective progestogens that enhance the beneficial (progestogenic) effects of the drug and reduce or eliminate the undesirable (mainly androgenic) effects like skin and hair changes, adverse effect on lipid profile ${ }^{4}$.

In 2001, FDA approved a new OCP that contains Drospirenone (DRSP) a novel progestin which is an analogue of spironolactone. It has pharmacological profile similar to Progesterone; particularly its antimineralocorticoid effects. It thus counteracts

\footnotetext{
Correspondence

Dr. Bhattacharya Parnamita, MS

Clinical tutor, Dept of Obs/Gyn

Calcutta Medical College and Hospital, Kolkata,India

Phone : 09830383036

Email : parnamita_bhattacharya@yahoo.co.in
} 
estrogen mediated fluid retention allowing stable or slightly lowered body weight. It also has an antiandrogenic effect on skin, hair, weight, lipid profile and beneficial effects on premenstrual symptoms ${ }^{5}$.

The aim of our study was to demonstrate the antiandrogenic and antimineralocorticoid benefits of drospirenone and to compare the changes in body weight, blood pressure, lipid profile, serum sodium and potassium level caused by drospirenone with desogestrel, widely used progesterone.

\section{Methods}

A prospective study was carried out in a tertiary medical Centre in eastern India from July2008 to June 2010. Two hundred women of 20-40 years age group attending the gynaecology outdoor for contraception or problems associated with polycystic ovarian disease, or those likely to be benefitted by non contraceptive advantage of OCP were included in the study.

After ruling out any contraindications of hormonal contraceptives, 100 subjects were administered ethinyl estradiol (30mcg) / drospirenone (3mg) combination and were referred to as DRSP/EE Group and 100 subjects were prescribed low dose oral contraceptive pills containing $30 \mathrm{mcg}$ ethinyl estradiol and $150 \mathrm{mcg}$ of desogestrol and referred to as other OCP group. Prior to therapy, body weight, blood pressure, lipid profile, serum sodium and potassium levels were measured, and scoring of hirsutism was done according to FG Score. Other causes of hirsuitism were excluded. Each individual were given the drug for 6 cycles.

Subjects were asked to come back after 1st cycle and at completion of therapy. Clinical examination was done and the initial investigations were repeated.

Antiandrogenic effect of drospirenone was studied by observing changes in FG score and lipid profile of the patients. The antimineralocorticoid activity of DRSP was studied by noting improvements in body weight, perimenstrual symptoms and changes in the serum sodium and potassium level.

\section{Results}

In this study most cases belonged to the age group of 20 to 25 years (51\%). Indication of prescribing the drugs in both groups is shown in table 1 and weight wise distribution of the cases are given in fig 1. Fifty nine percent of the total subjects did not have any hirsutism. 36 subjects in DRSP/EE group and 34 in the other group had mild hirsutism and 6 subjects each in both groups showed moderate hirsutism. None of the subjects had severe degree of hirsutism
Table 1. Indication of prescribing the drugs

\begin{tabular}{lcc}
\hline Indication & DRSP/EE & Other OCP \\
\hline Contraception & 40 & 42 \\
$\begin{array}{l}\text { Polycystic ovarian } \\
\text { syndrome }\end{array}$ & 28 & 30 \\
Dysmenorrhoea & 14 & 16 \\
Hirsutism & 10 & 8 \\
Functional ovarian cyst & 6 & 2 \\
Premenstrual tension & 2 & 2 \\
\hline \multicolumn{1}{c}{ Total } & $\mathbf{1 0 0}$ & $\mathbf{1 0 0}$ \\
\hline
\end{tabular}

\section{Side effects}

Nausea was the commonest side effect in EE/DRSP group; $16 \%$ compared to $18 \%$ in other OCP group. Breast tenderness was seen in only $10 \%$ of subjects in DRSP/EE group as compared to $20 \%$ in other OCP group. Only $6 \%$ of subjects on DRSP/EE had bloating as compared to $16 \%$ on other OCPs. $4 \%$ of subjects in DRSP group reported of headache and irritability whereas the incidence of headache and irritability was $8 \%$ and $6 \%$, respectively in the other OCP group.

In the DRSP/EE group 38(38\%) patients showed no change in their body weight at the end of 6 treatment cycles. Rest $62(62 \%)$ showed a small decrease in their body weight (statistically insignificant). In the study group receiving other low dose OCP 52 subjects showed increase in body weight, 32 had no change, and 16 had weight loss.

None of the subjects in DRSP/EE group had any increase in their FG score. Sixty eight patients showed no change and the rest $(32 \%)$ had a decrease in the FG score that is not clinically significant. In the other OCP group most of the patients (70\%), showed no change in their FG score, however it did not decrease in any of the cases. Thirty percent cases showed worsening of hirsutism clinically which is not statistically significant.

Majority of women in the study population had Systolic Blood Pressure (SBP) in the range of $>110-120 \mathrm{~mm}$ of $\mathrm{Hg}$ and diastolic blood pressure in the range of $>60$ to $70 \mathrm{~mm}$ of $\mathrm{Hg}$. In the group receiving DRSP/EE, $44(44 \%)$ subjects showed a decrease in systolic blood pressure and 36(36\%) subjects showed a decrease in diastolic blood pressure from baseline after completion of six cycles. None of the subjects showed any increase in blood pressure and all values were within normal limit. In the other OCP group none of the subjects 


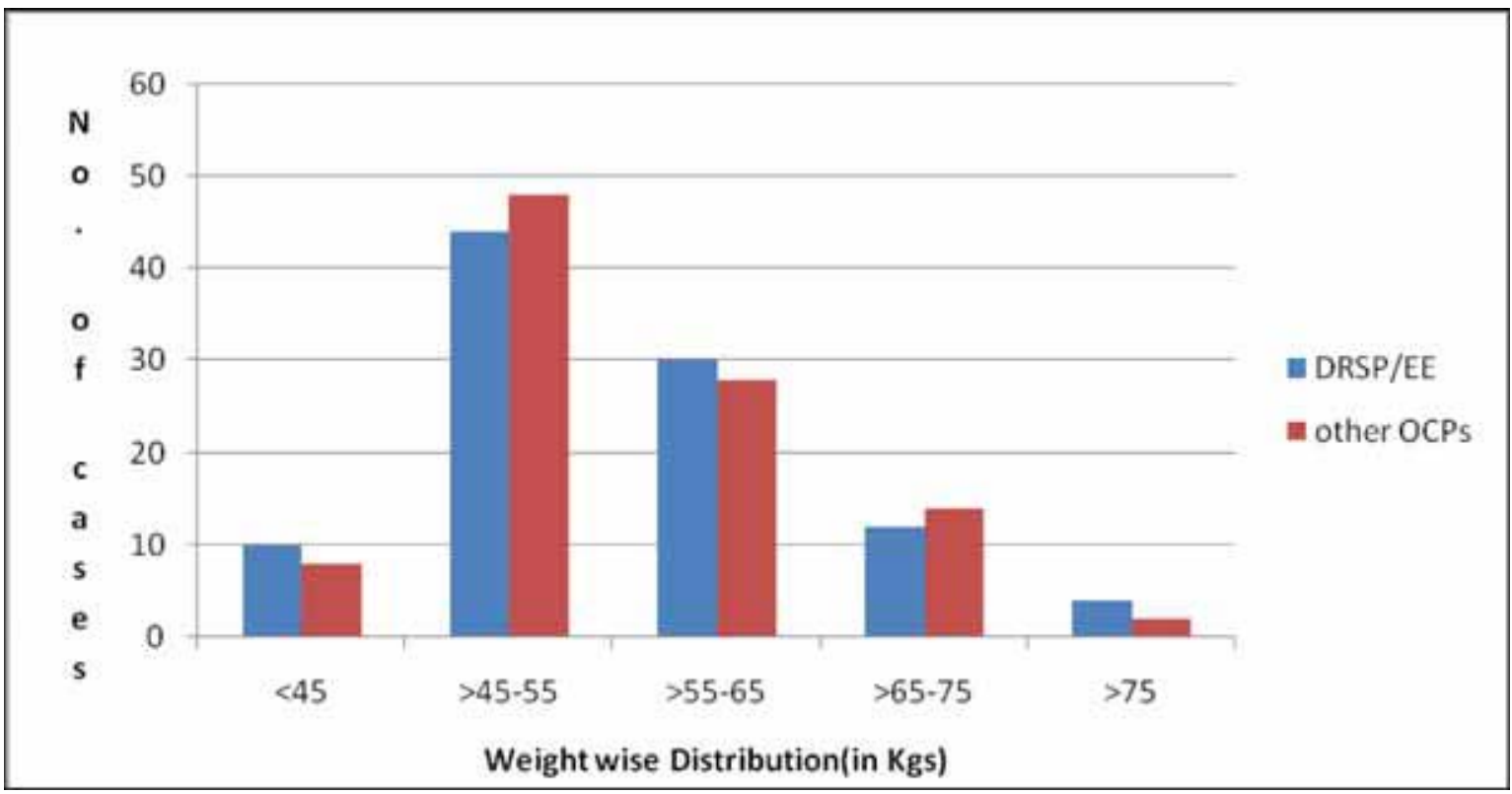

Table 2. Changes in serum sodium level after 6 cycles

\begin{tabular}{lcc}
\hline $\begin{array}{l}\text { Changes in serum } \\
\text { sodium level }\end{array}$ & $\begin{array}{c}\text { DRSP/EE group } \\
\text { (number of patients) }\end{array}$ & $\begin{array}{c}\text { Other OCP group } \\
\text { (number of patients) }\end{array}$ \\
\hline Increase & 0 & 62 \\
Decrease & 86 & 0 \\
No change & 14 & 38 \\
\hline Total & $\mathbf{1 0 0}$ & $\mathbf{1 0 0}$ \\
\hline
\end{tabular}

Table 3. Changes in the lipid profile in DRSP/EE group after six cycles

\begin{tabular}{lllll}
\hline \multicolumn{1}{c}{$\begin{array}{c}\text { LIPID } \\
\text { (mg/d) }\end{array}$} & \multicolumn{1}{c}{ Baseline } & \multicolumn{1}{c}{ After 6 Cycle } & \%Age change & \multicolumn{1}{c}{ p-Value } \\
\hline Serum Triglyceride & $158.3 \pm 26.1$ & $164.6 \pm 26.9$ & $\uparrow 3.97$ & Not significant \\
Serum HDL-C & $45.2 \pm 8.4$ & $48.6 \pm 8.7$ & $\uparrow 7.52$ & Significant $(\mathrm{p}=0.03)$ \\
Serum LDL-C & $59.5 \pm 13.1$ & $56 \pm 12.6$ & $\downarrow 5.88$ & Not significant \\
\hline
\end{tabular}

'able 4. Changes in lipid profile in the other OCP group after six cycles of therapy

\begin{tabular}{lcccc}
\hline \multicolumn{1}{c}{ LIPID ( mg/dl) } & Baseline & $\begin{array}{c}\text { After 6 cycles of } \\
\text { therapy }\end{array}$ & $\begin{array}{c}\text { Percentage } \\
\text { change }\end{array}$ & p-Value \\
\hline Serum Triglyceride & $153.7 \pm 24.7$ & $160.1 \pm 25.7$ & $\uparrow 4.16$ & Not significant \\
Serum HDL-C & $45.1 \pm 8.3$ & $48.3 \pm 9.6$ & $\uparrow 7.09$ & $\begin{array}{l}\text { Significant } \\
(\mathrm{p}=0.03)\end{array}$ \\
Serum LDL-C & $59.2 \pm 12.7$ & $61.7 \pm 13.6$ & $\uparrow 4.22$ & Not significant \\
\hline
\end{tabular}


showed any decrease in blood pressure. However $46(46 \%)$ subjects showed increase in Systolic blood pressure and 34(34\%) showed an increase in diastolic blood pressure.

At the beginning of the study, baseline serum sodium was in the range of $136-140 \mathrm{mmol} /$ litre $(47 \%)$ and serum potassium level in the range of $3.5-4 \mathrm{mmol} /$ litre. In the DRSP/EE group 86 subjects showed a decrease in the serum sodium level and rest 14 subjects had no change. All before and after values remained within normal limit. In the group administered other low dose OCPs 38 subjects showed no change in the serum sodium levels from the baseline and the rest 62 subjects showed a net gain in the serum sodium level (Table 2). Both the groups showed a rise in mean serum potassium values after 6 cycles but the mean level remained within normal range. All these changes had insignificant $\mathrm{p}$-values.

After 6 cycles, In the DRSP/EE group serum HDLCholesterol level increased by $7.52 \%$ and was statistically significant. Serum triglyceride increased by $3.97 \%$ and LDL-Cholesterol level decreased by $5.88 \%$ which is not statistically significant. Thus the changes in lipid profile were favourable. Women in other OCP group showed an increase in all three serum lipid values. Mean Serum triglyceride increases by $4.16 \%$, serum HDL-Cholesterol by $7.09 \%$ and serum LDL-Cholesterol by $4.22 \%$. Only the change in serum HDL-Cholesterol was clinically significant (Table 3, 4)

\section{Discussion}

In our study, there was a decrease in mean body weight by $1.25 \%$ in the DRSP/EE group and increase in mean weight by $0.69 \%$ from baseline in the other OCP group. Similar results were obtained in a study by Oelkers $\mathrm{W}$ et al, though the results of both studies were not statistically significant ${ }^{(6)}$. We also have found a statistically insignificant decrease in the FG score in $32 \%$ women in the DRSP/EE group and increase in none of them. No evidence of decrease in the FG score seen in any of the cases in other group whereas $30 \%$ of the subjects showed an increased value. Similar but statistically significant decrease in FG score was also evident with drospirenone in studies by Pehlivanov $\mathrm{B}$ et al, Guido $\mathrm{M}$ et al and Batukan $\mathrm{C}$ et $\mathrm{al}^{7,8,9}$ at 6 th cycle onwards and was maintained at $12^{\text {th }}$ cycle. In few previous studies as well as in our study, a general trend towards reduction in both systolic and diastolic blood pressure in the DRSP/EE group have been demonstrated, although these changes were not clinically significant at 6 months $s^{6,10}$.

This study showed that the subjects receiving DRSP/ EE either had no change in their serum sodium values or had statistically insignificant decrease in the values. This might be explained by the action of drospirenone on the renin angiotensin aldosterone system, thus preventing fluid and sodium retention in the body.

In the other OCP group, there was either an increase or no change in the serum sodium level. Probably because desogestrol does not counteract sodium and water retention caused by ethinyl estradiol. In a study by Oelker ${ }^{6}$ et al no significant change in serum sodium or potassium level occurred due to drospirenone /ethinyl estradiol or ethinyl estradiol/ levonorgesterol. Although more marked increase in aldosterone in the DRSP/ EE group reflected a compensatory natriuretic action.

In this study serum triglyceride showed a slight and insignificant increase in both the groups, reflecting the action of estrogen. Serum HDL-Cholesterol showed a significant increase in both the groups which is a known metabolic effect of both the estrogen and progestogen ${ }^{11}$. In the DRSP/EE group there was an insignificant decrease in serum LDL-cholesterol thus having a favourable impact on HDL-cholesterol/LDLcholesterol ratio as well as on cardiovascular system.

On the other hand in the other OCP group, LDLcholesterol showed a slight but non significant increase from baseline after 6 cycles, due to androgenic effect of desogestrol. In an open label multicentre study of 326 women who received DRSP (3mg)/EE (30 $\mu \mathrm{gm})$ for 12 cycles total cholesterol, triglyceride, HDL and HDL/LDL ratio showed similar but significant increase from baseline ${ }^{12}$.

\section{Conclusion}

The new progestin drospirenone has different pharmacological property and results in improved non contraceptive benefits and reduced side effects.

Its antimineralocorticoid activity prevents fluid retention, weight gain, bloating, rise in blood pressure and breast tenderness seen in some susceptible women with use of OCPs. Studies have also shown that it may produce a fall in both systolic and diastolic blood pressure. Drospirenone has an anti-androgenic effect at the receptor and enzymatic level, leading to reduction in appearance or worsening of hirsutism, oily skin and appearance of acne. These effect when seen with use other androgenic progestins is very distressing for women especially if they are young causing them to discontinue the pill.

Estrogen has a favourable effect on the lipid profile. Androgenic progestin attenuates this effect. Drospirenone being anti-androgenic, it decreases LDLcholesterol, increases HDL-Cholesterol and HDL/LDL ratio which reduces the atherogenicity of blood. 
Thus OCP containing drospirenone has positive effect on body weight, blood pressure, body fluid composition, and skin and hair changes and is a potentially well tolerated OCP for near future.

\section{References}

1. Fotherby K, Caldwel ADS. New Progestogens in oral contraception. Contraception 1994 Jan; 49:1-32

2. Bagshaw S. The combined oral contraceptive: risks and adverse effects in perspective. Drug Saf 1995 Feb; 12 (2): 91-6

3. Burkman RT, Shulman LP. Oral contraceptive practice guidelines. Contraception $1998 \mathrm{Sep} ; 58$ (3 Suppl.): 35S-43S.

4. Borgelt-Hansen L. Oral contraceptives: an update on health benefits and risks. J Am Pharm Assoc 2001 Dec; 41 (6): 875-86.

5. Mansour D. Yasmin-new oral contraceptive, a new progestogen: the reasons why. Eur J Contracept Reprod Health Care 2000 Dec; 5 (suppl. 3): 9-16.

6. Oelkers W, Foidart JM, Dombrovicz N, Welter A, Heithecker R. Effects of a new oral contraceptive containing an antimineralocorticoid progestogen, drospirenone, on the renin-aldosterone system, body weight, blood pressure, glucose tolerance, and lipid metabolism. J Clin Endocrinol Metab
$1995 ; 80: 1816-21$.

7. Pehlivanov B, Mitkov M. Efficacy of an oral contraceptive containing drospirenone in the treatment of women with polycystic ovary syndrome. Eur J Contracept Reprod Health Care 2007, 12(1): 30-5.

8. Guido M, Romualdi D, Giuliani M, Suriano R, Selvaggi L, Apa R, et al. Drospireonene for the treatment of hirsute women with polycystic ovary syndrome: a clinical, endocrinological, metabolic pilot study. J Clin Endocrinol Metab 2004:89: 2817-23.

9. Batukan C, Muderris II. Efficacy of a new oral contraceptive containing drospirenone and ethinyl estradiol in the long-term treatment of hirsutism. Fertill Sterill 2006, 85(2): 436-40.

10. Schurmann R, Holler T, Benda N. Estradiol and drospirenone for climacteric symptoms in postmenopausal women: a double-blind, randomized, placebo-controlled study of safety and efficacy of three dose regimens. Climacteric 2004; 7:189-96.

11. Mishell DR Jr. Cardiovascular risk: Perception versus reality. Contraception 1999, 59(1, Suppl.): 21S-4S.

12 Parsey KS, Pong A. An open - label, multicentre study to evaluate Yasmin ${ }^{(\mathrm{TM})}$ a low -dose combination oral contraceptive containing drospirenone, a new progestogen. Contraception 2000; 61: 105-11. 